\title{
Gamma-ray NLSy1s and 'classical' blazars: Are they different at radio $\mathrm{cm} / \mathrm{mm}$ bands?
}

\section{Lars Fuhrmann*}

Max-Planck-Institut für Radioastronomie Bonn, Germany

E-mail: Ifuhrmannempifr-bonn.mpg.de

\section{E. Angelakis, I. Nestoras, T. P. Krichbaum, N. Marchili, R. Schmidt, J. A. Zensus} Max-Planck-Institut für Radioastronomie Bonn, Germany

\author{
H. Ungerechts, A. Sievers, D. Riquelme \\ Institut de Radio Astronomie Millimétrique, Granada, Spain
}

L. Foschini, G. Ghisellini, G. Ghirlanda, G. Tagliaferri, F. Tavecchio, L. Maraschi INAF - Osservatorio Astronomico di Brera, Italy

\section{Giroletti}

INAF - Istituto di Radioastronomia, Bologna, Italy

\section{G. Calderone, M. Colpi}

Universita' di Milano-Bicocca, Dipartimento di Fisica, Milano, Italy

\section{R. Decarli}

Max-Planck-Institut für Astronomie, Heidelberg, Germany

\begin{abstract}
Since the early Fermi-GST discovery of NLSy1s as a new class of gamma-ray emitting AGN, we are monitoring the first source of this new class, J0948+0022, within a Fermi-dedicated blazar monitoring alliance called the F-GAMMA program: a sample of about 60 gamma-ray loud blazars is observed monthly with the Effelsberg 100-m and IRAM 30-m telescopes quasi-simultaneously at 10 frequencies between 2.6 and $142 \mathrm{GHz}$ ( $11 \mathrm{~cm}$ to $2 \mathrm{~mm}$ wavelength). In 2010, we extended the program towards two further Fermi-detected NLSy1s, namely $1 \mathrm{H} 0323+342$ and PKS 1502+036. Here, we present preliminary results of this first $\mathrm{cm} / \mathrm{mm}$ band monitoring of $\gamma$-ray, radio-loud NLSy1s with particular emphasis on their spectral and variability properties. Subsequently, we compare these properties with those obtained for the 'classical' Fermi-detected blazars in our sample and explore possible differences in their radio characteristics.
\end{abstract}

Narrow-Line Seyfert 1 Galaxies and their place in the Universe - NLS1,

April 04-06, 2011

Milan Italy

\footnotetext{
*Speaker.
} 


\section{Introduction}

Before the launch of Fermi-GST the known types of $\gamma$-ray emitting AGN were blazars and radio galaxies. Indeed, the early Fermi/LAT three-month results ([2]) confirmed that the extragalactic $\gamma$-ray sky is dominated by radio-loud AGNs, being mostly blazars and a few radio galaxies. However, an important and impressive early discovery of Fermi-GST is the detection of $\gamma$-rays from a different class of AGNs: Narrow Line Seyfert 1 galaxies (NLSy1s). These types of objects are believed to be active nuclei similar to those of Seyferts with optical spectra showing permitted lines from the broad-line region, although much narrower than typically those seen in Seyfert $1 \mathrm{~s}$ or blazars $\left(\operatorname{FWHM}(\mathrm{H} \beta)<2000 \mathrm{~km} \mathrm{~s}^{-1}\right)$. NLSy1s are generally hosted in spiral galaxies and furthermore appear to accrete with high Eddington ratios albeit exhibiting only low black-hole masses (e.g. [12]). These and other characteristics make NLSy1s a unique class of AGN, whereas as a class they are typically radio-quiet - only less than $7 \%$ (e.g. [14]) are found to be radio-loud (RL) and only $\sim 2.5 \%$ are found to be 'very' radio loud $\left(R_{S_{\text {radio }} / S_{\text {opt }}}>100\right)$.

The first Fermi/LAT detection of $\gamma$-rays from a NLSy1, namely in PMN J0948+0022 ([3]), certainly once more raised the question whether powerful relativistic blazar-like jets exist in this type of object, as indicated by previous studies in particular for the most radio-loud NLSy1 (e.g. [19]). The Fermi/LAT and MW observations of PMN J0948+0022 ([4]) clearly demonstrate for the first time the existence of a $\gamma$-ray emitting NLSy1 hosting a relativistic jet similar to blazars even though the environment in the vicinity of the central engine is most likely pretty different. However, this discovery strongly challenges the common paradigm that associates powerful relativistic jets with elliptical host galaxies.

However, despite being intensively studied at X-ray and optical bands, still little is known about the detailed, long-term radio properties of NLSy1s compared to 'classical' blazars as revealed by e.g. monitoring of variability, spectral evolution and VLBI morphology. Previous studies often used (low-frequency) radio surveys (e.g. NVSS, FIRST; 1.4 GHz), AGN catalogs and/or optical/Xray surveys (SDSS, ROSAT) to identify RL NLSy1 and/or 'very' RL NLSy1 samples (e.g. [16], [14], [19]) and often radio properties such as radio loudness, sizes and (non-simultaneous) variability and spectral indices have been deduced. A few VLA/VLBI studies have been performed revealing pc/kpc-scale source properties of NLSy1s (e.g. [18], [6], [11]).

In order to study the radio characteristics of ( $\gamma$-ray emitting) NLSy1s and therewith, investigate their possible jet emission in greater detail, a $\mathrm{cm} / \mathrm{mm}$ band NLSy 1 monitoring in total intensity and polarisation was initiated in 2009 as part of a larger, Fermi-GST related blazar monitoring program (F-GAMMA program, [10]). Here, we present for the first time radio $\mathrm{cm} / \mathrm{mm}$ monitoring (variability and broad band spectral evolution) results for NLSy1-type objects obtained using the Effelsberg 100-m and IRAM 30-m telescopes. Three Fermi-detected, 'very' radio-loud NLSy1s are continuously observed: J0324+3410, PMN J0948+0022 (hereafter J0948+0022) and J1505+0326. Preliminary results of this NLSy1 program are presented: the radio characteristics (broad band variability and spectral properties) are discussed, and finally compared to those of the 'classical', 'powerful-jet like' $\gamma$-ray blazars in our program. More details of this NLSy1 monitoring and the results will be presented subsequently (Fuhrmann et al., Angelakis et al. in prep.). 


\section{Observations}

Radio $\mathrm{cm} / \mathrm{mm}$ light curves and quasi-simultaneous broad band spectra for the three NLSy1s have been obtained within the framework of a Fermi-GST related monitoring program of $\gamma$-ray blazars ([10], [5]): the F-GAMMA (Fermi-GST AGN Multi-frequency Monitoring Alliance) program is the coordinated effort of several observatories and teams to probe AGN physics through the multi-frequency monitoring approach. The core program relies on monthly observations with the Effelsberg 100-m (EB) radio telescope (operating at 8 frequencies between 2.6 and $43 \mathrm{GHz}$ ), the IRAM 30-m telescope on Pico Veleta (PV, observing at 86 and $142 \mathrm{GHz}$ ) and additional measurements with the APEX 12-m telescope (operating at $345 \mathrm{GHz}$ ). The PV millimeter observations of the F-GAMMA program are closely coordinated with the more general flux monitoring conducted by IRAM, and data from both programs are included here. The core sample consists of roughly 60 Fermi-GST/LAT monitored blazars. The multi-frequency data of each source are taken simultaneously within 40 minutes for Effelsberg $(2.6-43 \mathrm{GHz})$ and truly simultaneous at the IRAM telescope (86 and $142 \mathrm{GHz}$ ) while the cross-station coherency is of order of $\lesssim 10$ days.

In June 2009, the Fermi-detected NLSy1 J0948+0022 was included in the program (EB and PV) after a major revision of the general source sample according to the first Fermi-LAT results ([2]) followed by the sources J0324+3410 and J1505+0326 in 2010 (EB only), after their FermiLAT detection ([1]).

\section{First Results and discussion}

\subsection{Light curves and $\mathrm{cm} / \mathrm{mm}$ band spectra}

In Fig. 1, first light curves of the three NLSy1s are shown including all data collected until 2011.2. Evidently, all three sources show some level of activity at basically all wavelengths, i.e. indicating variability on time scales of months to years.

The most pronounced variability is seen in the case of J0948+0022 (Fig. 1, center row) which exhibits the longest data sets $(2009.0-2011.2)$ as well as broadest frequency coverage including PV measurements at 86 and $142 \mathrm{GHz}$. The source was observed during a very active phase and shows a long-term increasing trend since $\sim 2009.3$ with several large amplitude, rapid "sub-flares" superimposed. The amplitude of the variability is increasing towards higher frequencies. Furthermore, flares appear delayed towards lower frequencies. The radio activity in 2010 happened during its $\mathrm{GeV}$ flaring activity - the first detected GeV flare of a NLSy1 with Fermi/LAT (see [7] for details).

For the NLSy1s J0324+3410 and J1505+0326 the observed time series are still too short (2010.6 - 2011.2) in order to obtain a detailed picture of their variability behavior. However, J0324+3410 (Fig. 1, top) shows a clear trend of monotonic decrease between $2010.6-2011.1$ at all EB bands followed by a pretty rapid increase until 2011.2. In the case of J1505+0326 the source appears less active during the observing period with no obvious stronger variations until 2010. However, a slight increase in flux density afterwards indicates possible pronounced variability/activity in the near future as well.

The obtained quasi-simultaneous broad band spectra are shown in Fig. 2. J0948+0022 shows dominant spectral evolution with mostly inverted spectra up to $43 \mathrm{GHz}$ and prominent spectral 

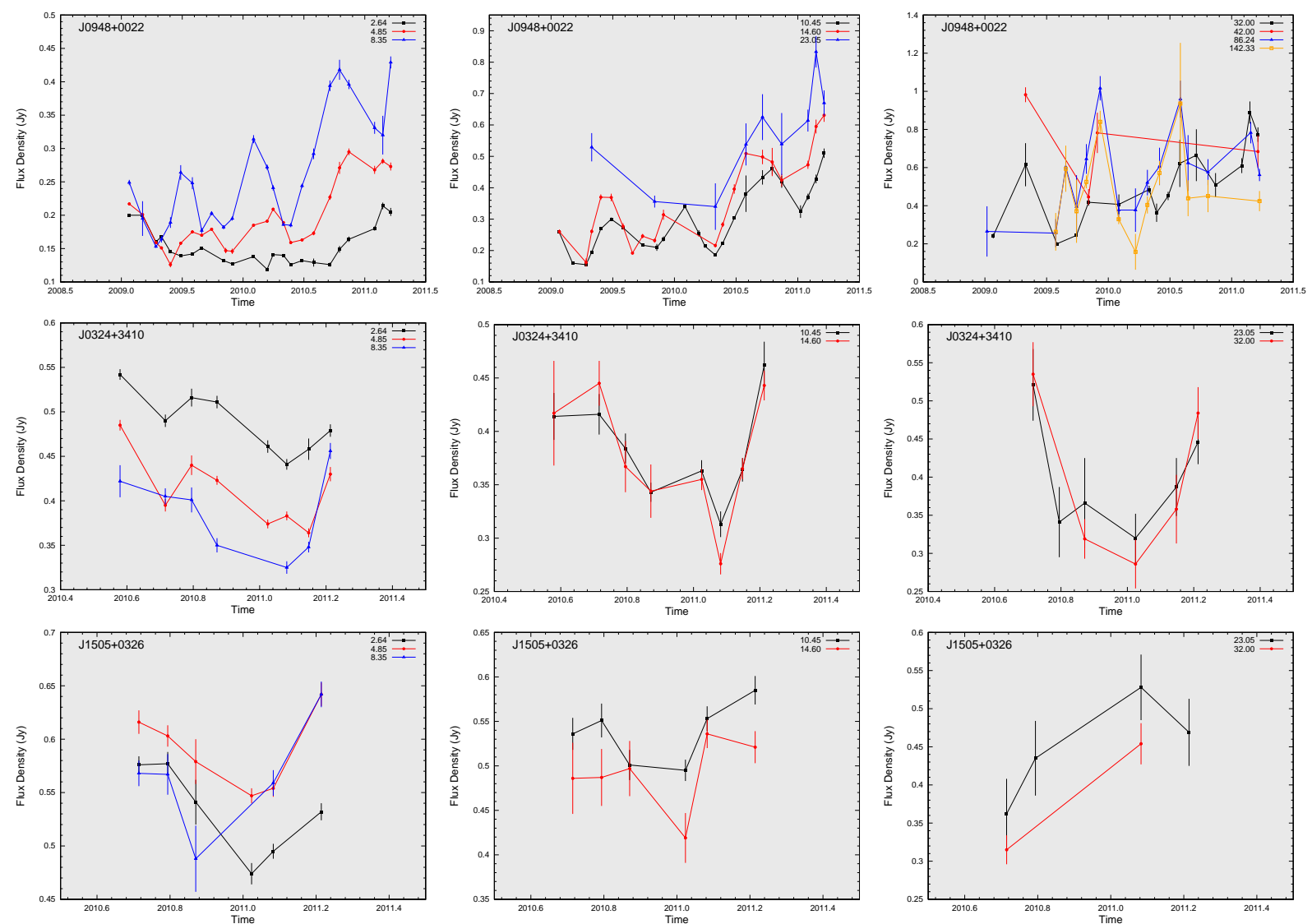

Figure 1: First multi-frequency light curves of the three $\gamma$-ray NLSy1s monitored by the F-GAMMA program. The inset labels give the different observing frequencies in GHz. Top: J0948+0022 with the longest time series as observed with EB and PV at low (left), medium (middle) and high (right) frequencies. Center: J0324+3410 as observed with EB at low (left), medium (middle) and high (right) frequencies. Bottom: J1505+0326 as observed with EB at low (left), medium (middle) and high (right) frequencies.

peaks occurring in the frequency range between that of EB and PV, i.e. between 43 and $86 \mathrm{GHz}$. Several 'events' are occurring which seem to move through the spectrum from the highest down to the lowest frequencies.

J0324+3410 exhibits a clear steepish spectral component starting at $2.6 \mathrm{GHz}$ with a flat/inverted spectral component temporarily appearing at the highest frequencies ( $\geq 10 \mathrm{GHz}$ ). J1505+0326 exhibits a flatish spectrum at frequencies less than $8 \mathrm{GHz}$ with a spectral steepening towards higher frequencies.

\subsection{Variability properties}

A first detailed variability analysis (see [9] for details) has been performed using the data shown in Fig. 1. A formal $\chi^{2}$-test reveals the presence of variability on a $99.9 \%$ significance level for J0948+0022 and J0324+3410 at all available frequencies. Due to the non-detection of significant variability at most of the observing bands, J1505+0326 is excluded from the following analysis. 

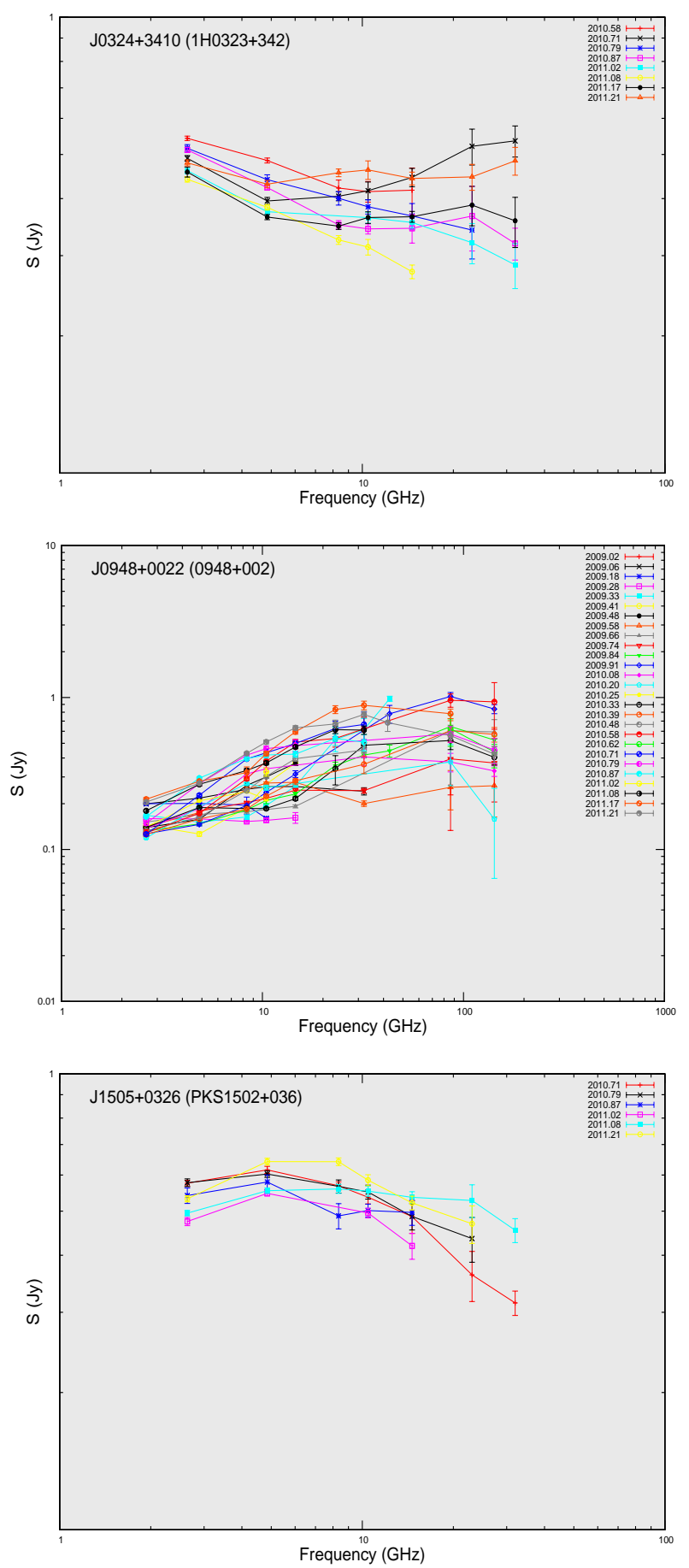

Figure 2: First $\mathrm{cm} / \mathrm{mm}$ broad band spectra of the three $\gamma$-ray NLSy $1 \mathrm{~s}$ monitored by the F-GAMMA program. The inset labels provide the observing epoch of each quasi-simultaneous spectrum. Top: J0324+3410 as observed with EB between 2.6 and $42 \mathrm{GHz}(11 \mathrm{~cm}$ to $7 \mathrm{~mm})$ Center: J0948+0022 as observed with EB and PV between 2.6 and $142 \mathrm{GHz}(11 \mathrm{~cm}$ to $2 \mathrm{~mm}$ ) Bottom: J1505+0326 as observed with EB between 2.6 and $42 \mathrm{GHz}$ (11 cm to $7 \mathrm{~mm}$ ) 
To further quantify the observed variability, the rms-variations and corresponding modulation indices $(m[\%]=100 \times \sigma /<S>$ ) of each light curve have been calculated. The results confirm the large variability amplitudes for J0948+0022 with $m$-values between $15.6 \%$ at $2.6 \mathrm{GHz}$ up to $45.8 \%$ at $142 \mathrm{GHz}$ (as comparison, typical values of non-variable calibrators are $0.8 \%$ at $2.6 \mathrm{GHz}$ and $3.0 \%$ at $32 \mathrm{GHz}$ ), i.e. the variability amplitude strongly increases towards higher frequencies. A similar behavior is observed in the case of J0324+3410 here, however, with lower amplitude values (between $7.6 \%$ and $35.6 \%$ at 2.6 and $32 \mathrm{GHz}$, respectively).

Finally, variability time scales of the multi-frequency light curves have been obtained using a detailed structure function and wavelet analysis. The obtained time scales and variability amplitudes furthermore provide estimates for variability source sizes, brightness temperatures $T_{B}$ and Doppler factors D (see e.g. [9] for details) using light-travel time arguments as well as the Inverse-Compton (IC) and/or equipartition brightness temperature limits ([13], [17]). The obtained variability time scales for J0948+0022 range between about 570 days (long-term trend) to events as short as about 90 days. The range of resulting brightness temperatures are $1.1 \times 10^{10} \mathrm{~K}(142 \mathrm{GHz})$ to $4.6 \times 10^{12} \mathrm{~K}(2.6 \mathrm{GHz})$ and Doppler factors $\leq 2.6$ are required to bring these values down to the theoretical IC limit. J0324+3410 exhibits variability time scales in the range of 60 and 180 days. Due to the so far limited observing period and thus only observed trends in the light curves, these values are only lower limits. The resulting $\mathrm{T}_{\mathrm{B}}$-values are in the range of $3.7-9.1 \times 10^{12} \mathrm{~K}$ and obtained Doppler factors $\leq 2.2$.

Thus, both sources appear relativistically Doppler boosted, however, only mild values are required in order not to violate the theoretical limits.

\subsection{Spectral properties}

Mean spectral indices $\left(S \propto v^{\alpha}\right)$ have been obtained from the quasi-simultaneous broad band spectra at low $(4.8,10,15 \mathrm{GHz})$ and for J0948+0022 also at high frequencies $(32,86,142 \mathrm{GHz})$. While $\mathrm{J} 0324+3410$ and $\mathrm{J} 1505+0326$ show on average flat spectra ( -0.08 and -0.18 , respectively) at low frequencies, J0948+0022 exhibits on average a highly inverted spectrum at both, low and high frequencies ( 0.43 and 0.21 , respectively), resembling the observed flaring activity of the source. The latter is also evident in significant evolution of the spectral peaks in the $S_{\max }-v_{\max }$ plane.

For the other two NLSy1s, the observing period is still to short to study their spectral evolution in detail. However, the low-level activity seen in their light curves of Fig. 1 seem to temporarily appear as 'up-turn' at the high frequency end of their spectra.

\subsection{Comparison to the 'classical' $\gamma$-ray bright blazars}

The variability and spectral properties of the three NLSy1s deduced above can now be compared to those obtained for the 'classical' $\gamma$-ray blazars of our sample to investigate possible differences in the two source classes. For this purpose, we use the data sets gathered over the first four years of observations (2007.0-2011.2, Angelakis et al., Nestoras et al. in prep.). Due to the length-limited data sets as well as statistically incomplete samples, we note however, that a detailed and robust statistical analysis is not possible.

The variability and spectral characteristics of the NLSy1s appear similar to those of the typical blazars: e.g. variability amplitudes increasing with frequency and mean spectral indices being flat 
to inverted. In particular, the inverted 'flare spectra' of J0948+0022 and corresponding evolution are similar to flaring events in our blazar sample showing prominent spectral evolution (Angelakis et al. in prep.) and adiabatic expansion related delays typical to those expected in a shock-injet scenario ([15]). Furthermore, the steep spectral component at low frequencies in J0324+3410 speaks in favor of the existence of an optically thin, 'quiescent jet' as often observed in blazar spectra.

However, differences seem to occur in terms of strength of variability, $T_{\mathrm{B}}$ and Doppler factors. Taking the rms-variations of the light curves, the NLSy1s exhibit much lower values than typically observed in the $\gamma$-ray blazar sample, i.e. they occur as the lower envelop when plotting rmsvariations vs. frequency including all sources (blazars and NLSy1s). J0948+0022, for which the longest light curves (with prominent events) are available, values differing by more than a factor of $\sim 100$ are seen.

In addition, the obtained brightness temperatures appear at the lower end of those typically observed in the blazar sample. For instance, the blazars' $T_{\mathrm{B}}$ values at $2.6 \mathrm{GHz}$ range between $4.0 \times$ $10^{11} \mathrm{~K}$ and $1.8 \times 10^{17} \mathrm{~K}$ (mean: $5.9 \times 10^{15} \mathrm{~K}$; median: $1.3 \times 10^{13} \mathrm{~K}$ ), while it is remarkable that the corresponding $T_{\mathrm{B}}$ of J0948+0022 is $4.6 \times 10^{12} \mathrm{~K}$. Similarly, at $4.8 \mathrm{GHz}$ we obtain a range of $8.5 \times$ $10^{10} \mathrm{~K}$ to $3.1 \times 10^{15} \mathrm{~K}$ (mean: $1.2 \times 10^{14} \mathrm{~K}$; median: $5.9 \times 10^{12} \mathrm{~K}$ ), and at $32 \mathrm{GHz}$ a range of $2.5 \times$ $10^{10} \mathrm{~K}$ to $1.4 \times 10^{13} \mathrm{~K}$ (mean: $2.1 \times 10^{12} \mathrm{~K}$; median: $1.2 \times 10^{12} \mathrm{~K}$ ) as compared to $4.0 \times 10^{11} \mathrm{~K}$ $(4.8 \mathrm{GHz})$ and $9.3 \times 10^{10} \mathrm{~K}(32 \mathrm{GHz})$ for J0948+0022, respectively. Similar results are obtained at other frequencies. Consequently, the resulting Doppler factors show the same behavior e.g. at 2.6 GHz, the blazars' mean is 8.7 (median: 4.1) as compared to a value of 2.6 for J0948+0022.

\section{Conclusions}

Preliminary results of the first long-term, $\mathrm{cm} / \mathrm{mm}$ monitoring program of NLSy1s have been presented providing - for the first time - broad band spectra and variability characteristics of (radioloud) NLSy1-type objects. The obtained variability and spectral characteristics of the first period of monitoring indicate blazar-like behavior, i.e. the three 'very' radio-loud $\gamma$-ray NLSy1s exhibit non-thermal synchrotron emission from relativistic jets seen close to the line of sight. Possible differences occur in variablity amplitudes, brightness temperatures and Doppler boosting compared to the 'classical' $\gamma$-ray blazars. The obtained lower values indicate intrinsically less powerful events occurring in NLSy1s and/or lower jet speeds or larger viewing angles towards these objects and hence, less Doppler boosting. Given the fact that the luminosity of J0948+0022 is observed to be similar to those of blazars (i.e. between those of flat-spectrum radio quasars and BL Lacs, [8]), it appears more likely that the viewing angles towards NLSy1s are larger than those towards blazars. However, longer time series are required in order to study such possible differences in greater detail.

\section{References}

[1] Abdo, A. A., et al. 2010, ApJ, 715, 429

[2] Abdo, A. A., et al. 2009, ApJ, 700, 597

[3] Abdo, A. A., et al. 2009, ApJ, 699, 976 
[4] Abdo, A. A., et al. 2009, ApJ, 707, 727

[5] Angelakis, E., et al. 2008, arXiv:0809.3912

[6] Doi, A., et al. 2006, PASJ, 58, 829

[7] Foschini, L., et al. 2011, MNRAS, 413, 1671

[8] Foschini, L., et al. for the Fermi/LAT Collaboration 2009, arXiv:0908.3313

[9] Fuhrmann, L., et al. 2008, A\&A, 490, 1019

[10] Fuhrmann, L., et al. 2007, The First GLAST Symposium, 921, 249

[11] Giroletti, M., et al. 2011, A\&A, 528, L11

[12] Grupe, D., \& Mathur, S. 2004, ApJ, 606, L41

[13] Kellermann, K. I., \& Pauliny-Toth, I. I. K. 1969, ApJ, 155, L71

[14] Komossa, S., et al. 2006, AJ, 132, 531

[15] Marscher, A. P., \& Gear, W. K. 1985, ApJ, 298, 114

[16] Moran, E. C. 2000, New Astronomy Reviews, 44, 527

[17] Readhead, A. C. S. 1994, ApJ, 426, 51

[18] Ulvestad, J. S., Antonucci, R. R. J., \& Goodrich, R. W. 1995, AJ, 109, 81

[19] Yuan, W., et al. 2008, ApJ, 685, 801 